\title{
Sistemas de producción de Spondias purpurea (Anacardiaceae) en el centro-occidente de México
}

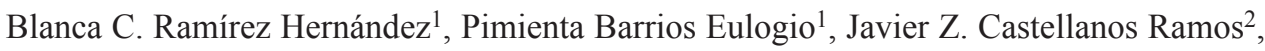 \\ Alejandro Muñoz Urias ${ }^{1}$, Guadalupe Palomino Hasbach ${ }^{3}$ \& Enrique Pimienta Barrios ${ }^{4}$ \\ 1. Departamento de Ecología. CUCBA Universidad de Guadalajara. México; bramirez@cucba.udg.mx. \\ 2. INIFAP-Región Centro. Campo Experimental Bajío. Celaya, Guanajuato, México. \\ 3. Jardín Botánico, Instituto de Biología. Universidad Nacional Autónoma de México. Ciudad de México. México \\ 4. Departamento de Producción Agrícola. CUCBA Universidad de Guadalajara. México.
}

\author{
Recibido 15-III-2007. Corregido 13-IX-2007. Aceptado 31-X-2007.
}

\begin{abstract}
Production systems of Spondias purpurea (Anacardiaceae) in Central West México. Morphological, physical and chemical traits related with fruit quality characteristics of Spondias purpurea L. agroecosystems were studied in Central-West Mexico for wild and cultivated populations. Spondias purpurea regularly thrive in shallow, rocky infertile soils unsuitable for conventional crops. The weight, axial and radial length, $\mathrm{pH}$, total soluble solids (SST), reducing sugars, proteins and mineral content in fruits were recorded. The mean fresh fruit weight was superior in the cultivated varieties $(20 \mathrm{~g})$ than in the wild $(16 \mathrm{~g})$. Similarly the highest values of $\mathrm{pH}$, SST, reducing sugars and protein content $\left(3.3,12.15^{\circ}\right.$ Brix, $0.38 \mathrm{~g} / 100 \mathrm{~g}$ and $1.18 \mathrm{~g} / 100 \mathrm{~g}$, respectively) were observed in the cultivated plantations compared with wild populations $\left(3.0,8.31^{\circ}\right.$ Brix, $0.24 \mathrm{~g} / 100 \mathrm{~g}$ and $0.14 \mathrm{~g} / 100 \mathrm{~g}$, respectively). In cultivated plantations, productivity ranged from 0.15 ton ha ${ }^{-1}$ to 5.0 ton ha- ${ }^{-1}$, and must be considered satisfactory, considering the low inputs of fertilizers and pesticides applied to orchards. The fruits of $S$. purpurea are similar in nutrimental content to more important commercial fruit species; it produces fresh fruits during the dry months of spring, when few fresh fruits are available in the local markets. In addition, $S$. purpurea is a source of water and food for domestic animals and wild fauna. These traits emphasize their agronomical and ecological importance for tropical and subtropical environments, where it can also be used in reforestation programs because it can grow in infertile rocky soils, and in agroecosystems inhabited by low income farmers that practice subsistence agriculture. In fact, the cultivation of Spondias has helped convert marginal lands into productive lands. Rev. Biol. Trop. 56 (2): 675-687. Epub 2008 June 30.
\end{abstract}

Key words: Spondias purpurea, Anacardiaceae, subtropical climate, agroecosystem description, fruit chemical composition.

Las especies de ciruela o jocote, Spondias spp., que producen frutos comestibles son originarias de América tropical, de las regiones del Pacífico y de algunos países asiáticos (Airy Shaw y Forman 1967, Morton 1987, Vázquez-Yanes et al. 1999, Macía y Barfod 2000, Miller y Schaal 2005). Únicamente dos especies de este género son nativas de México: Spondias purpurea L. y S. mombin L. var. mombin. Ambas se distribuyen desde México hasta Panamá junto con S. radlkoferi Donn. Sm. (Miller y Schaal 2005). En México se distribuye a lo largo de la costa occidental de
Sonora a Chiapas, y al sur del país en Yucatán y Quintana Roo y es un componente del estrato dominante de la selva baja caducifolia (Avitia 1996, Pennington y Sarukhán 1998, Miller y Schall 2005). S. purpurea presenta diversos centros de domesticación, uno de ellos abarca desde el sur de México hasta Panamá y el otro en la parte centro occidente de México (Miller y Schaal 2005). Los frutos de S. purpurea tienen amplia tradición de consumo en México desde la época prehispánica (Díaz del Castillo 1992; Benavente 1969, Turner y Miksicek 1984). En contraste con la mayoría 
de los alimentos usados por las culturas prehispánicas, el consumo de los frutos de Spondias se mantuvo en la época de la colonia (Benitez 1986). Las ciruelas se recolectaban de poblaciones silvestres en tiempos prehispánicos, y formaron parte de la dieta de los indígenas que poblaron las regiones tropicales de Veracruz (Díaz del Castillo 1992). El cultivo intensivo de $S$. purpurea en el Occidente de México se remonta al siglo XIX (Aldana 1986), y tuvo un auge al final del s. XIX, y al empezar el s. XX. Posteriormente $S$. purpurea, al igual que otras especies nativas, fue desplazada por la introducción de otros frutales de origen europeo, africano o asiático. Esta especie se encuentra distribuida o ha sido introducida a otros países de América y el mundo (Morton 1987, Crane y Campbell 1990, Macía y Barfod 2000), en donde a pesar de considerarse un fruto exótico con potencial para la exportación (Sturrock 1959, Leroy 1968, Samson 1986), ha tenido poco éxito económico, y regularmente los árboles de $S$. purpurea crecen en huertos familiares de pequeña extensión (Crane y Campbell 1990). En la actualidad los frutos se cosechan en poblaciones cultivadas y silvestres en diferentes regiones tropicales y subtropicales de México (Castro 1977, Rodríguez y Villaseñor 1988, Avitia et al. 2000, Ruenes y Jiménez 2001, Ramírez-Hernández 2004). Además de consumirse en fresco, las ciruelas también se emplean para elaborar diversos productos (e.g. mermeladas, bebidas, deshidratados) (LorenzoBautista 1977, Challenger 1998, Avitia, et al. 2000, Ramírez-Hernández 2004). El objetivo de este trabajo fue realizar la caracterización de algunos de los agroecosistemas en los que se cultiva $S$. purpurea en el Occidente de México, y describir las características físico-químicas de los frutos de las principales variedades silvestres y/o cultivadas que son aprovechadas por los habitantes de esta regiones

\section{MATERIALES Y MÉTODOS}

Localidades de estudio: Este trabajo se realizó en ocho localidades en poblaciones silvestres y/o cultivadas, en los estados de Jalisco,
Colima y Nayarit, en donde los frutos frescos se emplean para el autoconsumo y para comercializarlos en los mercados locales y regionales. Los sitios de estudio elegidos se localizan en una región considerada como uno de los centros domesticación de $S$. purpurea en el occidente de México (Miller y Schaal 2005). Las características geográficas y climáticas de las regiones estudiadas son descritas en la Cuadro 1.

La Barranca de Huentitán presenta clima subtropical subhúmedo (Medina-García et al. 1998). Los suelos son rocosos con pendiente mediana y baja fertilidad (Castro 1977), y se clasifican como regosoles, con un $\mathrm{pH}$ ligeramente alcalino (Rzedowski 1978, Galván 1988). En la zona de la Costa en el suroeste de Estado de Jalisco el clima es clasificado como semiseco con invierno fresco y primavera seca-cálida sin estación invernal definida (Medina García et al. 1998). En el estado de Colima, los suelos son clasificados en el orden de los afisoles y son pedregosos, poco profundos y arenosos (Estrada 1987). En la región Norte predominan dos tipos de clima el cálido subhúmedo y el semicálido subhúmedo. Los tipos de suelos que predominan son: cambizol, húmico, eútrico y dístrico; luvisol ortíco y crómico; acrisol ortíco; húmico feozen háplico; gleysol vértico, regosol eutríco y andosol húmico (Centro Nacional de Desarrollo Municipal 1999).

Estudio físico-químico de los frutos: Se recolectaron frutos de 12 variedades en las localidades en estudio. Por cada variedad fueron colectados 20 frutos en el estadio de madurez de consumo, en los que fue registrado: 1) peso fresco; 2) longitud axial y radial. Después fueron conservados a $-20^{\circ} \mathrm{C}$ en gas carbónico y llevados al laboratorio de Ecofisiología Vegetal de la Universidad de Guadalajara, donde fueron almacenados en un congelador a $-20^{\circ} \mathrm{C}$, para realizar análisis químicos en la pulpa, usando los procedimientos que se describen: 3 ) porcentaje de sólidos solubles totales (SST), se obtuvo con un refractómetro manual con temperatura compensada (Atago); 4) contenido de azúcares reductores. La extracción de azúcares 
CUADRO 1

Localidades de estudio de poblaciones silvestres y cultivadas de Spondias purpurea en el centro-occidente de México

\begin{tabular}{|c|c|c|c|c|c|}
\hline Región & Localidad & $\begin{array}{l}\text { Altitud } \\
(\mathrm{msnm})\end{array}$ & Coordenadas & $\begin{array}{c}\text { Temperatura } \\
\text { media anual }\left({ }^{\circ} \mathrm{C}\right)\end{array}$ & $\begin{array}{c}\text { Precipitación } \\
\text { pluvial anual }(\mathrm{mm})\end{array}$ \\
\hline \multirow{2}{*}{$\begin{array}{l}\text { Barranca de } \\
\text { Huentitán }\end{array}$} & $\begin{array}{l}\text { San Francisco de Ixcatán, } \\
\text { Jalisco }\end{array}$ & 1082 & $\begin{array}{c}103^{\circ} 20^{\prime} 23 ” \mathrm{~W} y \\
20^{\circ} 51^{\prime} 34^{\prime \prime} \mathrm{N}\end{array}$ & 25 & 906 \\
\hline & Paso de Guadalupe, Jalisco & 935 & $\begin{array}{c}103^{\circ} 19^{\prime} 37^{\prime \prime} \mathrm{W} \mathrm{y} \\
20^{\circ} 50^{\prime} 14^{\prime \prime} \mathrm{N}\end{array}$ & 25 & 906 \\
\hline \multirow{4}{*}{ Costa } & Cihuatlán, Jalisco & 400 & $\begin{array}{c}104^{\circ} 33^{\prime} 43^{\prime \prime} \mathrm{W} y \\
19^{\circ} 14^{\prime} 15^{\prime \prime} \mathrm{N}\end{array}$ & 26 & 967 \\
\hline & La Huerta, Jalisco & 450 & $\begin{array}{c}104^{\circ} 38^{\prime} 21^{\prime \prime} \mathrm{W} y \\
19^{\circ} 28^{\prime} 52^{\prime} \mathrm{N}\end{array}$ & 25 & 1.107 \\
\hline & Las Garzas, Colima & & $\begin{array}{c}104^{\circ} 17^{\prime} 56^{\prime \prime} \mathrm{W} \mathrm{y} \\
19^{\circ} 05^{\prime} 51^{\prime \prime} \mathrm{N}\end{array}$ & & \\
\hline & El Naranjo, Colima & 4 & $\begin{array}{c}104^{\circ} 24^{\prime} 58^{\prime \prime} \mathrm{W} \text { y } \\
19^{\circ} 07^{\prime} 40^{\prime \prime} \mathrm{N}\end{array}$ & 25 & 1.017 \\
\hline \multirow{2}{*}{ Norte } & El Jicote, Nayarit & 922 & $\begin{array}{c}105^{\circ} 03^{\prime} 33^{\prime \prime} \mathrm{W} y \\
21^{\circ} 43^{\prime} 23^{\prime \prime} \mathrm{N}\end{array}$ & 21 & 1.121 \\
\hline & Sauta, Nayarit & 900 & $\begin{array}{c}105^{\circ} 08^{\prime} 25^{\prime \prime} \mathrm{W} y \\
21^{\circ} 43^{\prime} 13^{\prime \prime} \mathrm{N}\end{array}$ & 26 & 1.316 \\
\hline
\end{tabular}

reductores se realizó utilizando la técnica de descrita por Carnal y Black (1989), modificada por Pimienta-Barrios y Nobel (1995). A $1 \mathrm{~g}$ de tejido se le agregaron $5 \mathrm{~mL}$ de etanol $(80 \%)$ para homogeneizarlo y se colocó en un baño de maría a $75^{\circ} \mathrm{C}$ por $5 \mathrm{~min}$. Posteriormente se centrifugó a $12000 \mathrm{~g}$ por $5 \mathrm{~min}$ y se colectó el sobrenadante y se combinó con el primero y se centrifugó a $27000 \mathrm{~g}$ por $20 \mathrm{~min}$ a $-4^{\circ} \mathrm{C}$. Posteriormente se colectó el sobrenadante y se aforó a un volumen de $25 \mathrm{~mL}$ con etanol al $80 \%$. De éste se tomaron alícuotas para evaluar el contenido de azúcares reductores utilizando el método de Somogy (1952); 5) contenido de proteína. Para la extracción de proteína se utilizó el método descrito por Choe y Thimann (1975), con algunas modificaciones. A 2 g de tejido se le agregaron $8 \mathrm{~mL}$ de acetona fría $(80 \%)$ para homogeneizarlo, se centrifugó a $12000 \mathrm{~g} \mathrm{a}-4^{\circ} \mathrm{C}$ durante $10 \mathrm{~min}$, se extrajo el sobrenadante. La operación anterior se repitió y al precipitado obtenido se le agregaron $8 \mathrm{~mL}$ de ácido tricloro-acético (10\%) frío y se almacenó a $-10^{\circ} \mathrm{C}$ por una hora. Posteriormente se agitó en un Vortex y se centrifugó a 12000 g por 20 min. Se descartó el sobrenadante y al precipitado se le adicionaron $3.2 \mathrm{~mL}$ de hidróxido de sodio (1 $\mathrm{N})$. La muestra se colocó en un baño de maría a $80^{\circ} \mathrm{C}$ por 20 minutos. Posteriormente se midió el volumen final. La proteína insoluble en acetona $(80 \%)$ se estimó utilizando el método de Lowry et al. (1951); 6) pH. Se registró con un potenciómetro (Conductronic $\mathrm{pH} \mathrm{20);7)}$ análisis de minerales. Las muestras de fruto se colocaron en una estufa de secado a $80^{\circ} \mathrm{C}$ hasta llevarlas a un peso constante. Posteriormente se pulverizaron en una moledora de café, y se tomaron $0.5 \mathrm{~g}$ de este pulverizado para digerirlo durante $12 \mathrm{~h}$ en $4 \mathrm{~mL}$ de ácido nítrico. Posteriormente se adicionaron $2 \mathrm{~mL}$ de ácido perclórico y después de una hora la mezcla se calentó a $170^{\circ} \mathrm{C}$ en una mufla. El contenido de minerales fue medido con el método colorimétrico empleando el método vanadate-molibdato (Jones et al. 1991); 8) porcentaje de agua. Para la realización de esta medición se tomó en cuenta la cáscara y la pulpa, el peso seco se obtuvo al colocar esta porción en una estufa de 
secado a $70^{\circ} \mathrm{C}$ hasta obtener un peso constante (después de 72 horas).

\section{RESULTADOS}

Agroecosistemas: En las regiones en estudio fueron registradas 12 variedades; 7 en poblaciones cultivadas y 5 en silvestres (Cuadro 2). En la región de la Barranca, es común que las poblaciones cultivadas $S$. purpurea se intercalen con otras especies de importancia económica como: guamuchil (Pithecellobium dulce), pitayo (Stenocereus queretaroensis) y mango (Mangifera indica); en la región de la Costa, con cítricos y mango, y en la región Norte con mango. Además, durante el periodo de lluvia (verano y parte del otoño) se dedican los espacios libres o calles para el cultivo de especies agrícolas anuales como maíz (Zea mays) y frijol (Phaseolus vulgaris), principalmente para autoconsumo.

Las plantaciones cultivadas son huertos de pequeña extensión (0.5-1.0 ha) localizados comúnmente en los traspatios de las poblaciones rurales y también como cerco vivo. El manejo de las plantaciones cultivadas en todas las localidades estudiadas es de baja intensidad. En pocos casos se aplican fertilizantes o pesticidas, y únicamente se realizan labores culturales de costo bajo (i.e. deshierbe, poda). La poda se efectúa después de la cosecha de los frutos y antes del establecimiento del periodo de lluvias, que es cuando inicia el crecimiento vegetativo (Pimienta-Barrios y Ramírez Hernández 2003). Los árboles cultivados se propagan asexualmente (estaca) y los silvestres por la vía sexual (semilla).

En la zona de la Barranca de Huentitán el cultivo de $S$. purpurea se incrementó notablemente desde mediados del siglo XX, y en el presente siglo es una de las zonas productoras más importantes en el Estado de Jalisco. En contraste, en la costa $S$. purpurea fue desplazada para cultivar otras especies que tienen mayor demanda en el mercado nacional e internacional (e.g. M. indica, Ananas comosus, Cocos nucifera) o para edificar fraccionamientos.
El rendimiento de frutos fue estimado registrando datos del número promedio de frutos por árbol; el promedio del peso de estos y la densidad de árboles por ha. En las localidades estudiadas el rendimiento varió de 0.15 ton $\mathrm{ha}^{-1}$, en árboles jóvenes (5 años de edad) a 1.5-3.0 ton $\mathrm{ha}^{-1}$ en árboles adultos (30-50 años), aunque se registraron plantaciones adultas con rendimientos de hasta 5.0 ton $\mathrm{ha}^{-1}$. La productividad en las poblaciones silvestres (por árbol) llega a ser similar al que se registra en árboles de plantaciones cultivadas; sin embargo, el rendimiento por superficie es menor, ya que en las silvestres es menor la densidad de árboles por ha. El ingreso promedio por temporada de cosecha en las localidades de estudio varió de 300 a 600 dólares americanos por hectárea ( 0.6 a 1.2 dólares americanos por $\mathrm{kg}$ ).

En las tres regiones de estudio los frutos se comercializan en los acotamientos de las carreteras, en tianguis o mercados regionales y recientemente se ha introducido su venta en las grandes cadenas de supermercados. Además de consumirse en fresco las ciruelas son utilizadas en la elaboración de bebidas, mieles, paletas de hielo, y algunas personas emplean el fruto como un ingrediente para elaborar platillos; únicamente en la región Norte los frutos se deshidratan para posteriormente hacer bebidas. Los frutos silvestres son más ácidos, y es común que estos se destinen para elaborar aguas frescas, alimentar al ganado o con fines medicinales. Las hojas del ciruelo también pueden ser consumidas y tienen aplicación medicinal.

Caracterización morfológica, física y química de frutos: El peso de los frutos de las variedades evaluadas varió de $6.3 \mathrm{~g}$ a 35.8 y el peso promedio fue más alto en un $19 \%$ $(20.0 \mathrm{~g})$ en las variedades cultivadas que en las silvestres $(16.0 \mathrm{~g})$. Una excepción fue la variedad silvestre "madura verde" en la que se registró el segundo mayor peso promedio $(27.0 \mathrm{~g})$; la variedad cultivada "tempranera" registró el peso promedio más bajo $(6.0 \mathrm{~g})$. La relación largo/ancho varió desde 0.74 a 0.91 , lo que indica la tendencia de estos frutos a ser globosos (Cuadro 3). 


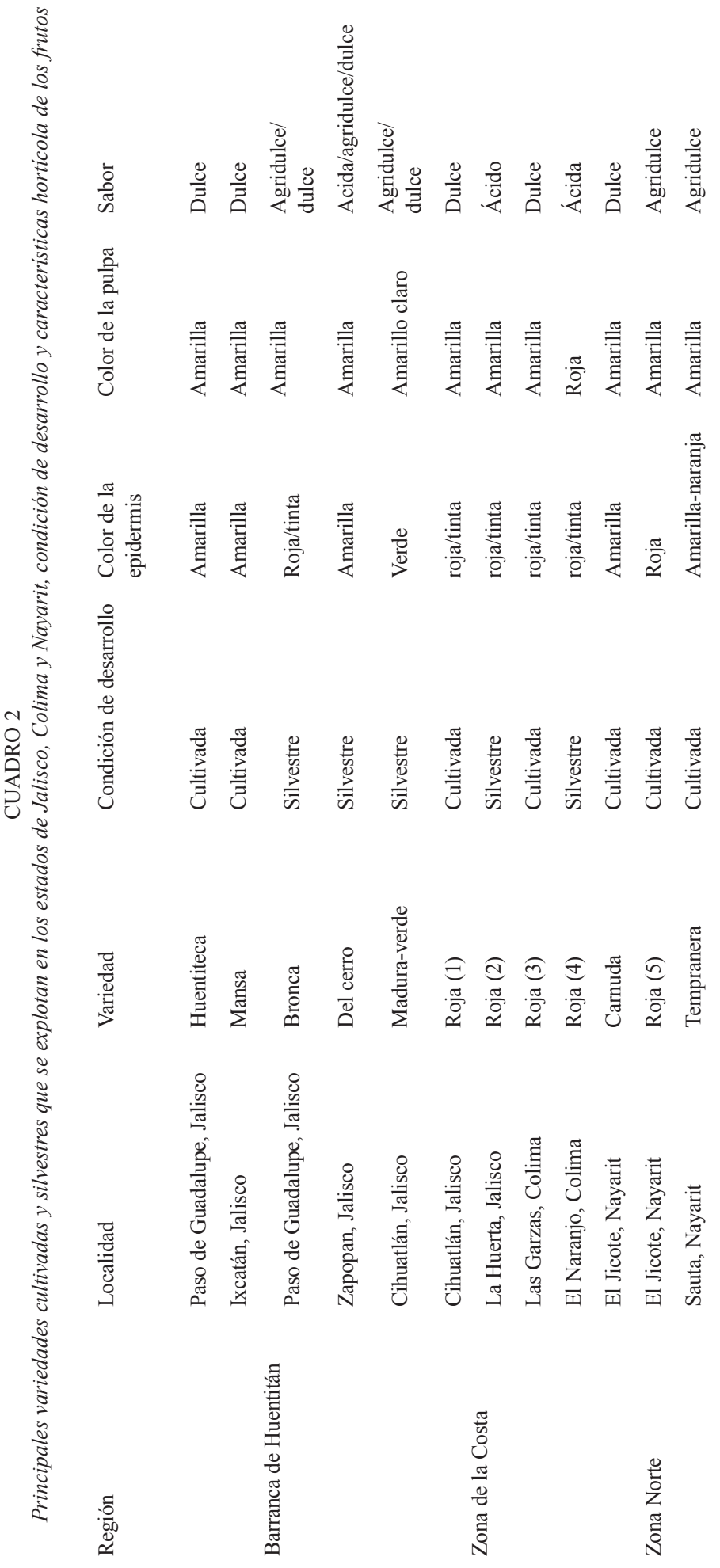


En la Cuadro 4 se muestra la composición química de los frutos de $S$. purpurea colectados en las regiones en estudio. El $\mathrm{pH}$ promedio varió de 2.7 a 3.5 , y este es más alto en las variedades cultivadas (3.3) que en las silvestres (3.0). El contenido de sólidos solubles totales varió de 7.0 a $15.6^{\circ}$ Brix, y fue más alto en las variedades cultivadas. Los valores de azúcares reductores oscilaron entre 0.11 y 0.52 $\mathrm{g} / 100 \mathrm{~g}$. El valor promedio de las variedades cultivadas $(0.38 \mathrm{~g} / 100 \mathrm{~g})$ resultó superior al de las silvestres $(0.24 \mathrm{~g} / 100)$. Los contenidos promedio de proteínas no mostraron diferencias significativas entre los frutos de las variedades silvestres $(0.16 \mathrm{~g} / 100 \mathrm{~g})$ y los de las cultivadas (0.15 g/100 g).

En la Cuadro 5 se observa el contenido de minerales en las diferentes variedades de ciruelas. En promedio el K, Fe y Mn fue superior en las variedades silvestres, mientras que el $\mathrm{N}, \mathrm{P} \mathrm{Cu}, \mathrm{Zn}$ fue superior en las cultivadas. No fueron registradas diferencias en el contenido de Mg. El contenido de fósforo en los frutos de las variedades cultivadas fue ligeramente superior $(2.0 \mathrm{mg} / 100 \mathrm{~g})$ al de las silvestres (1.9 $\mathrm{mg} / 100 \mathrm{~g}$ ); el de magnesio fue similar para variedades silvestres y cultivadas $(3.5 \mathrm{mg} / 100$ g). Este varió de 2.6 (bronca) a $4.6 \mathrm{mg} / 100 \mathrm{~g}$ (tempranera). El contenido promedio de zinc en frutos de variedades silvestres fue ligeramente superior $(1.31 \mathrm{mg} / 100 \mathrm{~g})$ al de las cultivadas $(1.27 \mathrm{mg} / 100 \mathrm{~g})$, encontrándose el menor contenido en la variedad silvestre bronca (1.03 $\mathrm{mg} / 100 \mathrm{~g})$ y el mayor $(1.77 \mathrm{mg} / 100 \mathrm{~g})$ en la variedad cultivada carnuda. El contenido promedio de manganeso y de hierro en general fue mayor para los frutos de variedades silvestres (Cuadro 4), sin embargo fue más alta la diferencia en el contenido de hierro.

\section{DISCUSIÓN}

La calidad de los frutos depende de las características morfológicas y físicas (i.e. color, tamaño, firmeza) y la composición química (i.e. relación azúcar/ácido, contenido de vitaminas y minerales) (Kushman y Ballinger 1975, Sistrunk y Moore 1988). La información proporcionada por los productores y consumidores, reveló diferencias contrastantes en la preferencia por los tipos de frutos de acuerdo al sabor, color, tamaño, y proporción de la porción comestible. Por ejemplo, en la región de la Barranca las poblaciones silvestres y cultivadas producen en mayor proporción frutos amarillos; en contraste, en la región Norte se producen frutos rojos y amarillos, y en la Costa hay preferencia por los frutos rojos. En estas dos últimas regiones hay una marcada preferencia por los frutos de mayor tamaño; estas diferencias encontradas en las variedades silvestres y cultivadas en el occidente de México probablemente sean un reflejo del uso de diferentes criterios para la selección de frutos durante el proceso de domesticación de S. purpurea (Miller y Knouft 2006), particularmente en la primeras etapas de este proceso (Bringhurst 1988).

No obstante que los frutos de $S$. purpurea son relativamente pequeños, su sabor, color, y valor nutrimental revelan que es un fruto con calidad aceptable en comparación con los frutos producidos por especies frutales más importantes en el mundo, ya que las ciruelas son una fuente importante de azúcares y minerales, y probablemente de ácido ascórbico, ya que comúnmente la acidez de los frutos es debido a la presencia de este ácido. En los frutos de $S$. purpurea se registraron valores de $\mathrm{pH}$ que variaron desde 2.7 a 3.5 , resultando inferiores a los observados en frutos como la manzana (Malus spp.) (pH 3.9; Bordeleau et al. 2002), la tuna (Opuntia spp.) (pH 6.4-7.1; PimientaBarrios 1990), pitaya (S. queretaroensis) ( $\mathrm{pH}$ 4.5: Pimienta-Barrios y Nobel 1995), pero son similares a los reportados para frutos de poblaciones cultivadas de $S$. purpurea en Ecuador (Koziol y Macía 1998), y superiores los reportados para S. mombin (Pinto et al. 2003; León y Shaw 1990).

El peso de los frutos registrado en $S$. purpurea es superior al reportado en Ecuador en variedades cultivadas (9.0 a 18 g) (Macía y Barford 2000), así como de otras especies como $S$. mombin, en la que se reporta un peso promedio de $12 \mathrm{~g}$, y con porcentaje más bajo de 
TABLA 3

Descripción morfológica y el porcentaje de porción comestible de los frutos de variedades de ciruela mexicana (Spondias purpurea L.) encontradas en los Estados de Jalisco, Colima y Nayarit. (Datos son promedio \pm desviación estándar)

\begin{tabular}{|c|c|c|c|c|c|c|}
\hline \multirow[b]{2}{*}{ Variedad } & \multirow[b]{2}{*}{ Población } & \multicolumn{4}{|c|}{ Longitud } & \multirow{2}{*}{$\begin{array}{c}\text { Porción } \\
\text { comestible } \\
(\%)\end{array}$} \\
\hline & & $\begin{array}{l}\text { Peso } \\
(\mathrm{g})\end{array}$ & Radial (cm) & $\begin{array}{l}\text { Axial } \\
(\mathrm{cm})\end{array}$ & $\begin{array}{c}\text { Relacion largo/ } \\
\text { ancho }\end{array}$ & \\
\hline Mansa & Cultivada & $36 \pm 1.8$ & $3.46 \pm 0.27$ & $4.23 \pm 0.13$ & 0.81 & 85.00 \\
\hline Madura-verde & Silvestre & $27 \pm 1.7$ & $3.6 \pm 0.30$ & $4.21 \pm 0.31$ & 0.85 & 77.58 \\
\hline Roja (1) & Cultivada & $27 \pm 2.6$ & $3.27 \pm 0.25$ & $3.57 \pm 0.19$ & 0.91 & 76.84 \\
\hline Carnuda & Cultivada & $21 \pm 2.8$ & $2.69 \pm 0.23$ & $3.06 \pm 0.32$ & 0.87 & 78.73 \\
\hline Roja (5) & Cultivada & $17 \pm 1.5$ & $3.00 \pm 0.22$ & $3.50 \pm 0.26$ & 0.86 & 74.43 \\
\hline Roja (3) & Cultivada & $17 \pm 2.4$ & $3.06 \pm 0.13$ & $3.47 \pm 0.19$ & 0.88 & 73.80 \\
\hline Bronca & Silvestre & $16 \pm 5.3$ & $2.85 \pm 0.26$ & $3.43 \pm 0.27$ & 0.83 & 76.47 \\
\hline Roja (2) & Silvestre & $14 \pm 0.8$ & $2.59 \pm 0.17$ & $3.47 \pm 0.22$ & 0.74 & 71.73 \\
\hline Huentiteca & Cultivada & $13 \pm 2.5$ & $2.84 \pm 0.22$ & $3.12 \pm 0.20$ & 0.91 & 81.58 \\
\hline Roja (4) & Silvestre & $12 \pm 1.3$ & $2.73 \pm 0.14$ & $3.24 \pm 0.13$ & 0.84 & 69.76 \\
\hline Del cerro & Silvestre & $9 \pm 0.8$ & $2.40 \pm 0.24$ & $2.70 \pm 0.20$ & 0.88 & 83.23 \\
\hline Tempranera & Cultivada & $6 \pm 0.6$ & $2.72 \pm 0.11$ & $3.13 \pm 0.16$ & 0.86 & 65.86 \\
\hline
\end{tabular}

\section{TABLA 4}

Valores en la composición química de la pulpa de frutos colectados en poblaciones silvestres y cultivadas de diferentes variedades de Spondias purpurea L. en Jalisco, Colima y Nayarit. (Datos son promedio \pm desviación estándar)

$\begin{array}{llcccc}\text { Variedad } & \text { Población } & \mathrm{pH} & \begin{array}{c}\text { Sólidos solubles totales } \\ \left({ }^{\circ} \text { Brix }\right)\end{array} & \begin{array}{c}\text { Azúcares reductores } \\ (\mathrm{g} / 100 \mathrm{~g})\end{array} & \begin{array}{c}\text { Proteínas } \\ (\mathrm{g} / 100 \mathrm{~g})\end{array} \\ \text { Huentiteca } & \text { Cultivada } & 3.3 \pm 0.30 & 15.6 \pm 0.41 & 0.52 \pm 0.05 & 0.24 \pm 0.01 \\ \text { Mansa } & \text { Cultivada } & 3.3 \pm 0.35 & 13.3 \pm 0.90 & 0.51 \pm 0.16 & 0.11 \pm 0.01 \\ \text { Carnuda } & \text { Cultivada } & 3.4 \pm 0.40 & 13.0 \pm 1.21 & 0.40 \pm 0.63 & 0.10 \pm 0.05 \\ \text { Roja (1) } & \text { Cultivada } & 3.3 \pm 0.29 & 12.0 \pm 1.54 & 0.29 \pm 0.05 & 0.41 \pm 0.04 \\ \text { Bronca } & \text { Silvestre } & 3.3 \pm 0.30 & 10.8 \pm 0.66 & 0.33 \pm 0.09 & 0.16 \pm 0.05 \\ \text { Roja (3) } & \text { Cultivada } & 3.5 \pm 0.45 & 11.0 \pm 1.06 & 0.17 \pm 0.10 & 0.10 \pm 0.04 \\ \text { Tempranera } & \text { Cultivada } & 3.3 \pm 0.51 & 9.5 \pm 0.83 & 0.28 \pm 0.15 & 0.58 \pm 0.07 \\ \text { Madura-verde } & \text { Silvestre } & 3.1 \pm 0.64 & 8.5 \pm 1.62 & 0.11 \pm .098 & 0.07 \pm 0.14 \\ \text { Roja (5) } & \text { Cultivada } & 3.1 \pm 0.31 & 8.0 \pm 1.12 & 0.37 \pm 0.08 & 0.11 \pm 0.12 \\ \text { Del cerro } & \text { Silvestre } & 3.1 \pm 0.47 & 7.5 \pm 1.35 & 0.43 \pm 0.62 & 0.10 \pm 0.02 \\ \text { Roja (2) } & \text { Silvestre } & 3.0 \pm 0.38 & 7.5 \pm 0.63 & 0.18 \pm 0.07 & 0.16 \pm 0.04 \\ \text { Roja (4) } & \text { Silvestre } & 2.7 \pm 0.30 & 7.0 \pm 0.89 & 0.16 \pm 0.12 & 0.23 \pm 0.06\end{array}$




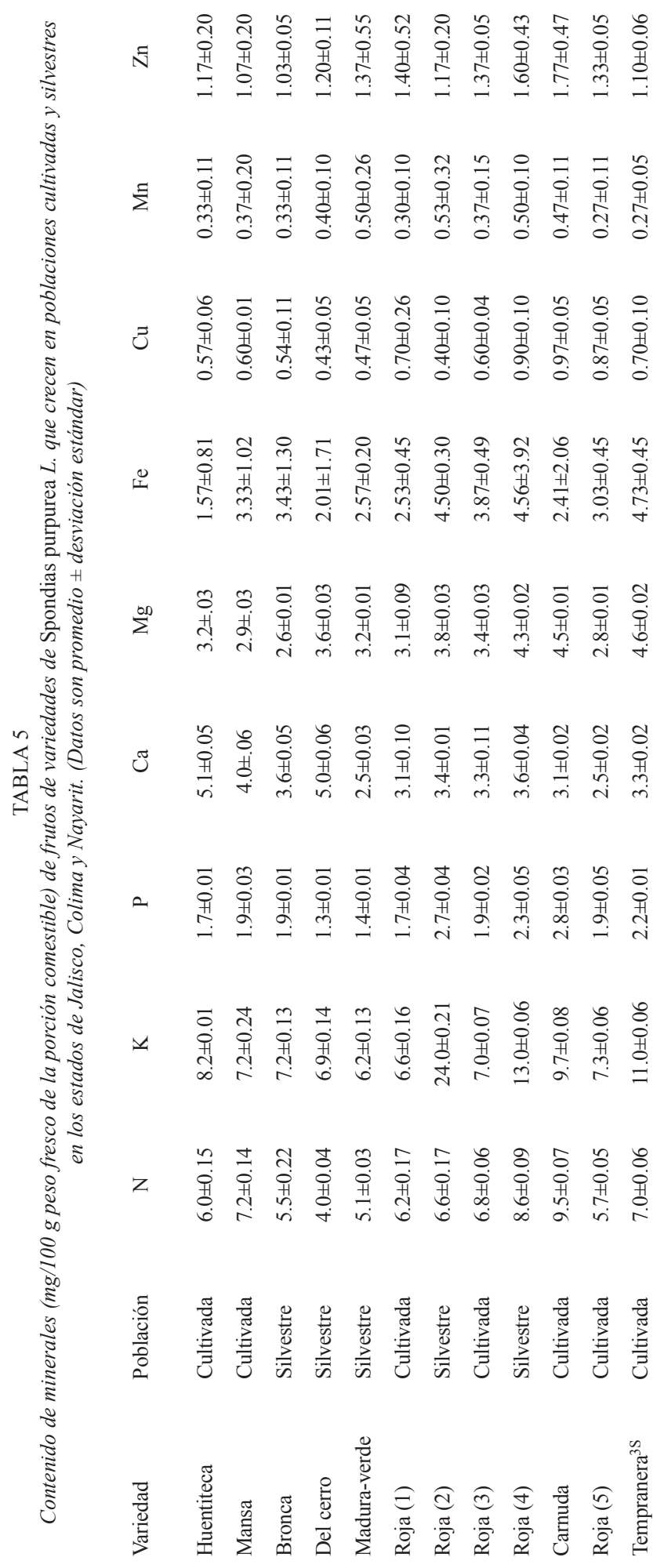


porción comestible (49.96\%; Pinto et al. 2003). Miller y Knouft (2006) refieren que el cultivo de $S$. purpurea a partir de árboles silvestres ha llevado a incrementar ciertos atributos de los frutos, sin embargo, en otras especies frutales nativas de México que se han sometido a domesticación, como es el caso de algunas cactáceas productoras de frutos comestibles (Opuntia spp. y Stenocereus spp.) han presentado incrementos hasta de más de $300 \%$ en el peso del fruto durante su domesticación (Tomas 1992, Pimienta-Barrios 1994, Pimienta-Barrios y Nobel 1994).

En cuanto a los sólidos solubles totales, la mayoría de las variedades cultivadas de $S$. purpurea de las regiones de estudio presentaron valores superiores al $12^{\circ}$ Brix por lo que se consideran de calidad nutrimental aceptable, ya que los frutos considerados de alta calidad presentan valores superiores a $11^{\circ}$ Brix (Williams y Cifuentes 1992), y son similares a los de la mayoría de los frutos frescos de las especies frutales más importantes. Por ejemplo, la variedad "huentiteca" presentó valores superiores a la manzana (Bordeleau et al. 2002), litchi (Litchi chinensis), uva (Vitis spp.), granada (Punica granatum), mango (Wills et al. 1998); tuna (Tomas 1992) y pitaya (Pimienta-Barrios y Nobel 1994), e incluso superior a la de otras especies del mismo género como $S$. mombin (Pinto et al. 2003, Owolarafe et al. 2006) y $S$. tuberosa (Santos 1997).

En relación al contenido de proteínas, las ciruelas presentan valores inferiores a los reportados en otras poblaciones cultivadas de la misma especie $(0.7$ g/100; Koziol y Macía 1998); León y Shaw (1990) reportan valores de 0.096, 0.261 hasta $0.9 \mathrm{~g} / 100$. Para S. mombin se reportan valores superiores de 0.8 a $1.3 \mathrm{~g} / 100 \mathrm{~g}$ y para $S$. dulcis 0.5 a $0.8 \mathrm{~g} / 100 \mathrm{~g}$ (León y Shaw 1990). Con relación a otras especies frutales, el contenido de proteína es ligeramente inferior a la guayaba (Psidium guajava) $(0.3 \mathrm{~g} / 100 \mathrm{~g})$, papaya (Carica papaya) y mango $(0.39 / 100$ g), así como a Ananas comosus $(0.5 \mathrm{~g} / 100 \mathrm{~g})$ (Nakasone y Paull 1998), también se reportan valores superiores a $1 \mathrm{~g} / 100 \mathrm{~g}$ para uvas, naranjas (Citrus spp.); otros frutales reportan cifras superiores por ejemplo plátano (Musa paradisiaca) $1.7 \mathrm{~g} / 100 \mathrm{~g}$ (Wills et al. 1998), aguacate (Persea americana) (1.8 g/100 g) (Salunkhe y Kadam 1995). Los valores que más se acercan a los reportados para $S$. purpurea son para manzana $(0.2 \mathrm{~g} / 100 \mathrm{~g})$ (Salunkhe y Kadam 1995) y para Pasiflora edulis (2.2 g/100 g) (Lamberts y Crane 1990).

La ciruela, al igual que otros frutos comestibles, es una fuente importante de minerales. El contenido promedio de calcio para frutos de las variedades cultivadas fue ligeramente inferior $(3.5 \mathrm{mg} / 100 \mathrm{~g})$, que el obtenido para las variedades silvestres $(3.6 \mathrm{mg} / 100 \mathrm{~g})$. El contenido de calcio en frutos de $S$. purpurea se puede equiparar con frutales que tienen un grado de domesticación avanzado; manzana $4 \mathrm{mg} / 100 \mathrm{~g}$, plátano $5 \mathrm{mg} / 100 \mathrm{~g}$ (Wills et al. 1998) (Cuadro 5), mango $16 \mathrm{mg} / 100 \mathrm{~g}$ de porción comestible (Owen et al. 1999), y superior si lo comparamos con frutos exóticos de reciente introducción en el mercado internacional, por ejemplo en $P$. edulis cuyo contenido de calcio es de 3.6 y $1 \mathrm{mg} / 100 \mathrm{~g}$ en carambola (Averrhoa carambola) (Nakasone y Paull 1998).

Uno de los factores que probablemente ha impedido la aceptación en el mercado internacional de $S$. purpurea es su corta vida en anaquel. Por lo tanto la comercialización de los frutos frescos se da a nivel local y regional al igual que en otras especies del género Spondias (i.e. S. tuberosa Arruda, S. mombin L.; Campbell 1996), y en algunos frutos tropicales y subtropicales. Los frutos que se producen en ambientes cálidos, regularmente son más delicados durante el manejo post-cosecha que los de especies frutales que se producen en climas frescos-templados (Burdon 1997). El procesamiento de los frutos, como ha sucedido con otras especies Spondias, podría ser una alternativa de consumo y comercialización para $S$. purpurea y reducir la limitante de corta vida en anaquel de los frutos (St. Louis y Badrie 2002).

En el occidente de México la maduración de los frutos de $S$. purpurea habita en la época seca el año por lo que difiere con la fenología de otras variedades de la especie en diferentas 
regiones del resto del país (Ruenes y Jiménez 2001), y puede ser considerada como una especie frutal estratégica para la agricultura de subsistencia en el centro-occidente de México, debido a que pocas especies producen frutos frescos en la primavera (periodo seco del año) y los frutos pueden cotizarse a precios relativamente altos. Además, debe resaltarse su relevancia ecológica, debido a que en la primavera se reduce la disponibilidad de agua y alimento para la fauna residente y migratoria, $S$. purpurea puede proveer agua y alimento para esta fauna (Mandujano et al. 1994), y también para el ganado doméstico (Ramírez-Hernández 2004).

Las diferencias de los factores ambientales y climatológicos (i.e. radiación, temperatura media anual, precipitación) en los que la especie se desarrolla (Miller y Knouft 2006), además de las diferencias existentes en los patrones fenológicos nos habla de la plasticidad de esta especie para responder a recursos disponibles, como es el caso de nutrimentos del suelo y la luz (Lambers et al. 1998, Aerts y Chapin III 2000, Ryser y Eek 2000, Arntz y Delph 2001, Ramírez-Hernández 2004), lo cual es relevante dado el ambiente en el que esta especie se desarrolla. Otra ventaja de esta especie es que, además de tener rápido crecimiento, se propaga vegetativamente, por lo que a corto plazo puede servir para reducir la erosión del suelo y recuperar el ciclo de nutrimentos (Barnes et al. 1998). De esta forma, $S$. purpurea puede emplearse como parte de las estrategias para restaurar ambientes tropicales degradados (Vázquez-Yanes et al. 1999, NavaCruz et al. 2007). De hecho, el cultivo de esta especie puede ayudar a convertir suelos marginales en suelos productivos, particularmente suelos pedregosos de baja fertilidad con pendientes pronunciadas, ya que presenta desarrollo y productividad satisfactoria en suelos no aptos para la agricultura convencional.

\section{AGRADECIMIENTOS}

EsteestudiofueauspiciadoporlaUniversidad de Guadalajara, y el Programa de Mejoramiento del Profesorado (PROMEP-SEP). Se agradece la asesoría Botánica de Raymundo Ramírez Delgadillo del Departamento de Botánica y Zoología del CUCBA. Los autores agradecen a los revisores anónimos y al editor sus valiosas sugerencias para mejorar el documento.

\section{RESUMEN}

Describimos las características de los agroecosistemas y rasgos morfológicos, físicos y químicos relacionados con la calidad de los frutos de la guayaba, Spondias purpurea L., que se producen en poblaciones silvestres y cultivadas en el centro-occidente de México. Spondias purpurea se desarrolla en suelos rocosos, poco profundos e infértiles, poco aptos para la agricultura tradicional. En los frutos se registró el peso, longitud axial y radial, $\mathrm{pH}$, sólidos solubles totales (SST), azúcares reductores, proteínas y contenido de minerales. El peso promedio de frutos en las variedades cultivadas $(20 \mathrm{~g})$ resultó superior al de las silvestres $(16 \mathrm{~g})$. Las variedades cultivadas registraron en promedio los valores más altos de $\mathrm{pH}, \mathrm{SST}$, azúcares reductores y contenido de proteínas $\left(3.3,12.15^{\circ}\right.$ Brix, $0.38 \mathrm{~g} / 100 \mathrm{~g}$ y $1.18 \mathrm{~g} / 100$ $\mathrm{g}$, respectivamente) que las silvestres $\left(3.0,8.31^{\circ}\right.$ Brix, 0.24 $\mathrm{g} / 100 \mathrm{~g}$ y $0.14 \mathrm{~g} / 100 \mathrm{~g}$, respectivamente). La productividad de la especie es aceptable (de 0.15 ton $\mathrm{ha}^{-1}$ a 5.0 ton $\mathrm{ha}^{-1}$ ) dado que requiere de un mínimo de manejo agronómico. Esta especie tiene un contenido nutrimental comparable al de las especies frutales más importantes; además, produce frutos frescos durante los meses secos de la primavera por lo que $S$. purpurea es fuente de agua y alimento para la fauna silvestre y doméstica. Estas características resaltan su importancia agronómica y ecológica para los ambientes tropicales y subtropicales en donde puede ser utilizada en programas de reforestación por su capacidad para desarrollarse en suelos rocosos infértiles, y en agroecosistemas en los que se practica agricultura de subsistencia. De hecho, el cultivo de $S$. purpurea puede ayudar a convertir suelos marginales en productivos.

Palabras clave: Spondias purpurea, Anacardiaceae, clima subtropical, composición química de frutos, agroecosistemas.

\section{REFERENCIAS}

Aerts, R. \& F.S. Chapin III. 2000. The mineral nutrition of wild plants revisited: A re-evaluation of processes and patterns, p. 1-67. In H.A. Fitter \& D.G. Raffaelli (eds). Advances in Ecological Research. Academic Press, San Diego, California, Estados Unidos de Norteamérica.

Airy Shaw, H.K. \& L.L. Forman. 1967. The genus Spondias L. (Anacardiaceae) in tropical Asia. Kew Bulletin 21: $1-20$. 
Aldana, R.M. 1986. El campo Jalisciense durante el porfiriato. Instituto de Ciencias Sociales. Universidad de Guadalajara, Guadalajara, México, 168 p.

Arntz, A. M. y L. F. Delph. 2001. Pattern and process: evidence for the evolution of photosynthetic traits in natural populations. Oecologia 127:455-467.

Avitia, G.E. 1996. Anatomía precigótica y postcigótica en relación al aborto de óvulos y semillas en Spondias purpurea L. Tesis de Doctorado. Colegio de Postgraduados. Montecillo, México. 118 p.

Avitia, G.E., A.M. Castillo G. \& E. Pimienta-Barrios. 2000. Ciruela mexicana y otras especies del género Spondias L. Universidad Autónoma de Chapingo. Chapingo, México. 75 p.

Barnes, B. V., D. R. Zak, S. R. Denton \& S.H. Spurr. 1998. Forest Ecology. Wiley. Nueva York, Estados Unidos de América. 774 p.

Benavente, Fray T. 1969. Historia de los Indios de la Nueva España. Porrúa. México D.F., México. 256 p.

Benitez, F. 1986. La ruta de Hernán Cortés. Fondo de Cultura Económica. México D.F., México 308 p.

Bordeleau, G., I. Myers-Smith, M. Midak \& A. Szeremeta. 2002. Food Quality: A comparison of organic and conventional fruits and vegetables. Ecological Agriculture, Den Kongelige Veterinær- og Landbohøjskole. Dinamarca. 82 p.

Bringhurst, R.S. 1988. Estrategia genotécnica, p.197-205. In J.N. Moore \& J. Janick (eds.). AGT Métodos genotécnicos en frutales. México D.F., México.

Burdon, J.N. 1997. Postharvest handling of tropical and subtropical fruit for export, p. 1-19. In Sisir Mitra (ed.). Postharvest Physiology and Storage of Tropical and Subtropical Fruits. CAB International. Londres, Inglaterra.

Campbell, R.J. 1996. South American Fruits deserving further attention, p. 431-439. In J. Janick (ed.). Progress in New Crops. ASHS, Arlington, Virginia, Estados Unidos de América.

Carnal, W.C. \& C.C. Black. 1989. Soluble sugars as the carbohydrate reserve for CAM in pineapple leaves. Plant Phisiol. 90: 91-100.

Castro, A.Z. 1977. Cultivo del ciruelo (Spondias spp.), en el municipio de San Cristóbal de la Barranca, Jalisco. Tesis de Licenciatura. Escuela de Agronomía. Universidad de Guadalajara. Guadalajara, Jalisco, México. 110 p.
Centro Nacional de Desarrollo Municipal. 1999. Enciclopedia de los Municipios de México: Colima. Gobierno del Estado de Colima. Colima, México.

Challenger, A. 1998. Utilización y conservación de los ecosistemas terrestres de México. Pasado, presente y futuro. Comisión Nacional para el Conocimiento y Uso de la Biodiversidad. México, D.F., México. 847 p.

Choe, T.H. \& K.V. Thimann. 1975. The metabolism of oat leaves during senescence III. The senescence of isolated chloroplast. Plant Physiol. 55: 828-834.

Crane, J.H. \& W. Campbell. 1990. Origin and distribution of tropical and subtropical fruits, p. 1-65. In S. Nagy \& P. Shaw, W. Wardoski (eds.). Fruits of Tropical and Subtropical Origin: Composition, Properties and Uses. Florida Science Source, Inc. Lake Alfred, Florida, Estados Unidos de América.

Díaz del Castillo, B. 1992. Historia verdadera de la conquista de la Nueva España. Editores Mexicanos Unidos. 2da edición. México D.F., México. 771 p.

Estrada, V.R.I. 1987. El cultivo del nance (Byrsonima crassifolia L.) en el Ejido de Campos, Mpio de Manzanillo, Colima. Tesis Ing. Agrónomo. Fac. de Agronomía. Universidad de Guadalajara. Guadalajara, México. 75 p.

Galván, R.R. 1988. Los municipios de Jalisco. Colección Enciclopédica de los Municipios de México. Secretaria de Gobernación y Gobierno del Estado de Jalisco, Centro Nacional de Estudios Municipales de la Secretaría de Gobernación, México D.F., México. $837 \mathrm{p}$.

Jones, Jr. J.B., B. Wolf \& H.A. Mills 1991. Plant analysis handbook: a practical sampling preparation, analyses and interpretation guide. Micro Macro Intl., Athens, Georgia, Estados Unidos de América. 422 p.

Koziol, M.J. \& M.J. Macía. 1998. Chemical composition, nutritional evaluation and economic prospects of Spondias purpurea (Anacardiaceae). Econom. Bot. 52: 373-380.

Kushman, L.J. \& W.E. Ballinger. 1975. Relation of quality indices of individual blueberries to photoelectrics measurements of anthocyanin content. J. Amer. Soc. Hort. Sci, 100: 561-564.

Lambers, H., S.F. Chapin III \& T.L. Pons. 1998. Plant physiological ecology. Springer Verlag, Nueva York, Estados Unidos de América. 540 p.

Lamberts, M. \& H.H. Crane. 1990. Tropical fruits, p. 337-355. In J. Janick \& J.E. Simon (eds.). Advances 
in New Crops. Timber, Portland, Oregon, Estados Unidos de América.

León, J. \& P Shaw. 1990. Spondias: The red mombin and related fruits, p. 116-126. In Nagy S. \& P. Shaw, W. Wardoski (eds.). Fruits of Tropical and Subtropical Origin: Composition, Properties and Uses. Florida Science Source, Inc. Lake Alfred, Florida, Estados Unidos de América.

Leroy, J.F. 1968. Les fruits tropicaux et subtropicaux. Press Universitaires de France. París, Francia. 128 p.

Lorenzo-Bautista, J.L. 1977. El desarrollo prehistórico e histórico de los agroecosistemas, p. 1-21. In Hernández-Xolocotzi, E. (ed.). Agroecosistemas de México. Colegio de Postgraduados. Primera Edición. Chapingo, México.

Lowry, O.H., N.J. Roseborough, A.L. Farr \& R.J. Randall 1951. Protein measurements with the folin phenol reagent. J. Biol. Chem. 193: 265-275.

Macía, J.M. \& A.S. Barfod. 2000. Economic botany of Spondias purpurea (Anacardiaceae) in Ecuador. Econom. Bot. 54: 449-458.

Mandujano, S., S. Gallina \& S.H. Bullock. 1994. Frugivory and dispersal of Spondias purpurea. Rev. Biol. Trop. 42:107-114.

Medina-García, G., J.A. Ruiz-Corral \& R.A. MartínezParra. 1998. Los Climas de México. CIRPC-INIFAPSAGAR. Libro Técnico No. 1. Guadalajara, Jalisco, México. 103 p.

Miller, A. \& B. Schaal. 2005. Domestication of a Mesoamerican cultivated fruit tree, Spondias purpurea. P.N.A.S. 102: 12801-12806.

Miller, A.J. \& Knouft, J.H. 2006. GIS-based characterization of the geographic distributions of wild and cultivated populations of the Mesoamerican fruit tree Spondias purpurea (Anacardiaceae). Am. J. Bot. 93: 1757-177.

Morton, J. 1987. Purple mombin, p. 242-245. In Julia F. Morton (ed.). Fruits of Warm Climates. Creative Resource Systems, Inc. Miami, Florida, Estados Unidos de América.

Nakasone, HY. \& R. E. Paull. 1998. Tropical fruits. CAB International, Wallingtonford, Reino Unido. $445 \mathrm{p}$.

Nava-Cruz, Y., M. Maass-Moreno, O. Briones-Villareal \& I. Mendez-Ramirez. 2007. Evaluation of the edge effect on two arboreal species of the tropical dry forest of Jalisco, Mexico. Agrociencia 4: 111-120.
Owen, A.L., P.L. Splett \& G.M. Owen. 1999. Nutrition in the community: the art and science of delivering services. Fourth ed. Mc.Graw-Hill. Boston, Estados Unidos de América. 654 p.

Owolarafe, O.K., Adebooye, O.C. \& Adegbenjo, O.A. 2006. Physical properties and food value of Spondias mombin L. - an underexploited fruit of Nigeria. J. Food Sci. Technol. Mysore. 43: 626-628.

Pennington, T.D. \& J. Sarukhán. 1998. Árboles tropicales de México. 2da edición. Fondo de Cultura Económica, Universidad Nacional Autónoma de México. México D.F., México. 521 p.

Pimienta-Barrios, E. 1994. Prickly pear (Opuntia spp.): a valuable fruit crop for the semiarid lands of Mexico. J. Arid Env. 27: 1-11.

Pimienta-Barrios, E. \& B.C. Ramírez-Hernández. 2003. Phenology, growth, and response to light of ciruela mexicana (Spondias purpurea L. Anacardiaceae). Econom. Bot. 57: 481-490.

Pimienta-Barrios, E. \& P.S. Nobel. 1994. Pitaya (Stenocereus spp. Cactaceae): an ancient and modern fruit crop of Mexico. Econom. Bot. 48: 76-83.

Pimienta-Barrios, E. \& P.S. Nobel. 1995. Reproductive characteristics of pitayo (Stenocereus queretaroensis) and their relationship with soluble sugars and irrigation. J. Amer. Soc. Hort. Sci. 120: 1082-1086.

Pimienta-Barrios, E. 1990. El nopal tunero. Libros Tiempos de Ciencia. Departamento de Investigación Científica y Superación Académica. Universidad de Guadalajara, Guadalajara, Jalisco, México. 246p.

Pinto, W.S., A.C.V.L. Dantas, A.A.O. Fonseca, C.A.S. Ledo, S.C. Jesus, P.L.P. Calanfage \& E. M. Andrade. 2003. Caracterizacao física, físico-química e química de frutos de genótipos de cajazeiras. Pesq. Agropec. Bras. 38: 1059-1066.

Ramírez Hernández, B.C. 2004. Etnobotánica y ecofisiología de ciruela mexicana (Spondias purpurea L.). Tesis Doctoral. Universidad Nacional Autónoma de México. México D.F., México. 113 p.

Rodríguez, E. S. \& H. S. Villaseñor. 1988. Evaluación de la propagación por estacado de tres tipos de ciruela mexicana (Spondias spp L.) en el estado de Colima, p. 133. In Resúmenes del XII Congreso de Fitogenética 18-22 de julio 1988. Universidad Autónoma de Chapingo. Chapingo, México.

Ruenes, M.R. \& J. Jiménez. 2001. Phenologycal characterization of Spondias purpurea L. (Anacardiaceae) cultivated in the home gardens of Hocabá, Yucatán. 
The $21^{\text {st }}$ Symposium in Plant Biology. "Lowland Maya Area: Three Millenia at the Human-Wild land Interface. Universidad de California, Riverside, California, Estados Unidos de América.

Ryser, P. \& L. Eek. 2000. Consequences of phenotypic plasticity vs. interespecific differences in leaf and root traits for acquisition of aboveground and belowground resources. Amer. J. Bot. 87: 402-411.

Rzedowski, J. 1978. Vegetación de Mexico. Limusa Noriega, Mexico D.F., México. 432 p.

Salunkhe, D.K. \& S.S. Kadam. 1995. Handbook of fruit science and technology: production, composition, storage and processing. CRC, Nueva York, Estados Unidos de América. 611 p.

Samson, J.A. 1986. Tropical fruits. Longman Scientific \& Technical, Singapur. 335 p.

Santos, C.A.F. 1997. Dispersão da variabilidade fenotípica do umbuzeiro no semi-árido brasileiro. Pesq. Agropec. Bras. 32: 923-930.

Sistrunk, W.A. \& J.M. Moore. 1988. Calidad, p. 367-395. In J. N. Moore \& J. Janick (eds.). Métodos Genotécnicos en Frutales. AGT, S. A. México, D.F., México.

Somogyi, M. 1952. Notes of sugar determination. J. Biol. Chem. 195: 19-23.

St. Louis, C. \& N. Badrie. 2002. Production of a fruit based hot sauce using golden apples (Spondias cytherea) in the Caribbean: Effects of peel addition, brining and storage on quality sauces. J. Food Quality. 25: 519-532.

Sturrock, D. 1959. Fruits for southern Florida. Southeastern Printing, Florida, Estados Unidos de América. 186 p.

Tomas, V.M.L. 1992. Caracterización de la variación de algunos componentes químicos de la pulpa y semilla del fruto de pitaya (Stenocereus queretaroensis [Weber] Buxbaum). Tesis de licenciatura, Universidad de Guadalajara, Guadalajara, Jalisco, México. 86 p.

Turner, B.L. \& C.H. Miksicek. 1984. Economic plant species associated with Prehistoric agriculture in the Maya lowlands. Econom. Bot. 38: 179-93.

Vázquez-Yanes, C., A.I. Bastis-Muñoz, M.I. Alcocer-Silva, M. Gual-Díaz \& C. Sánchez-Dirzo. 1999. Árboles $\mathrm{y}$ arbustos nativos potencialmente valiosos para la restauración ecológica y la reforestación. Reporte técnico del proyecto JO84. CONABIO. Instituto de Ecología, UNAM. http://xolo.conabio.gob-mx/arboles/intret-JO84.ntml.

Williams, W.T. \& S. Cifuentes. 1992. Rejuvenecimiento de un huerto modelo de melocotón en el Altiplano de Guatemala por medio de manejo integrado, p. 69-81. In G. Fischer \& F. Torres C. (eds.). International Symposium on Fruit Growing in Tropical Highlands. Acta Horticulturae no. 310. Bogotá, Colombia.

Wills, R., B. McGlasson, D. Graham \& D. Joyce. 1998. Postharvest: An introduction to the physiology \& handling of fruit, vegetables \& ornamentals. CAB International, Sidney, Australia. 262 p. 